\title{
Noise and Performances Analysis of Commerical Aircrafts using Artificial Neural Networks
}

\author{
Şahin YILDIRIM \\ Mechatronics Engineering Department \\ Erciyes University, Engineering Faculty \\ Kayseri-Turkey \\ sahiny@erciyes.edu.tr
}

\begin{abstract}
Commerical aircrafts are very important part for airway travelling. In spite of high technology on aircrafts, there is still fatality accidents in the world. Because of this reason, it is very important criteria to analyse noises of main elements of the air-craft systems. In tis study, an aircraft's main disturbances are analysed with proposed neural networks. Firstly, the noises of the jet, turbine and fan were measured from the aircraft. Secondly, the measured parameter values were predicted the proposed neural networks. The results of the proposed neuarl approaches were shown that this type of neural predictors will be employed to predict aircrafts unpredicted disturbances in real time applications.
\end{abstract}

Keywords: Neural predictor; noise; turbibe; fan; jet of aircraft

\section{I.INTRODUCTION}

The potential to reduce noise at source is limited and landuse measures are difficult to implement in densely populated zones. Operational procedures which depend on pilot behavior may lead to a reduction in the level of flight safety. The growth of air traffic is faster than developments in new technologies and methods of noise reduction.

Local environmental airport capacity can be expressed in terms of the maximum numbers of aircraft, passengers and freight accommodated during a given period under a particular environmental limitation and consistent with flight safety $[1,2]$

At present, only 2 per cent of the population is exposed to aircraft noise. This proportion should be compared with, for example, the 45 per cent of the population exposed to noise of road traffic and the 30 per cent to industrial noise. Neverthless, ICAO analysis has suggested that there will be a 42 per cent increase in the number of people affected by aircraft noise in Europe by the year $2020[3,4]$.

The noise produced by aircraft during operations in the areas around airports represents a serious social, ecological, technical and economic problem. Substantial levels of noise emission can bring about worsening of people's health, lowering their quality of life and lessening their productivity at work, through speech interference for example. In the areas around airports, aircraft noise has adverse influences on ground, maintenance and flight operations personnel, on passengers and on the local residential population.

Although aircraft are not the only sources of environmental noise around airports, they are the main ones. The working cycle of aircraft can be subdivided into starting engine operation, preflight engine run, taxiing to lineup, acceleration on the runway with full or reduced throttle, takeoff and rollon, flight path, landing, run-on operation and engine run-up. The maximum noise levels are made during the acceleration on the runway, takeoff and roll-on. But these stages are of relatively short duration. Other periods of aircraft noise generation around an airport occur during engine testing, maintenance work, temporary repair and engine replacement after the end of their service life. Maintenance operations and engine run-ups have a long duration and take place at comparatively short distances in relation to surrounding residential zones, passengers and technical staff. So, although they involve lower levels than those from moving aircraft, noise from these ground operations must be considered [5].

\section{MAIN DISTURBANCES OF AIRCRAFT AND DESCRIPTION}

Aircraft are complex noise sources (see Fig. 1). So a variety of noise protection methods are employed around airports; including organizational, technical, operational and zoning methods. The main noise sources on an aircraft in flight are the power unit and the aerodynamic noise. Aerodynamic noise becomes particularly noticeable during the landing approach of heavy jet aircraft, when the engines are at comparatively low thrust. The scientific basis for abating noise from aircraft relies on advances that have been made in aeroacoustics. Unlike classical acoustics (which is concerned mainly with the sound caused by oscillating surfaces), aeroacoustics investigates aerodynamic noise conditioned by turbulent nonstationary flow. Typically, jet aircraft noise sources include: jet noise, core noise, inlet and aft fan noise, turbine noise and airframe noise. Usually third-octave band spectra are used for noise assessment of any type of aircraft in any mode of flight or during maintenance activities in the vicinity of the airport. 
In this case, the common computational procedure for the prediction of the aircraft noise under the flight path or around the aircraft on a ground (run-ups, taxiing, waiting for the takeoff along the runway) is based on the assumption that sound waves are spreading along the shortest distance between the aircraft and the point of noise control [6].

\section{A. The main sources of aircraft noise}

There has been a considerable decrease in noise levels from individual aircraft over the past 35 years. The noise levels produced by modern aircraft are about $22 \mathrm{~dB}$ lower than those of first generation jet aircraft. This reduction has been achieved as a result of the development of turbofan engines with high bypass ratios, liner technology and turbomachinery source noise reduction. Further aircraft noise reduction will be achieved from improved design of engine systems, decrease in airframe sources and the introduction of noise reduction technologies

\section{1) Jet noise}

The characteristics of noise from a turbofan engine depend upon its construction and the parameters of flow in the duct of the engine. There are many methods and computer programs for predicting aviation noise[6-15]. Here semi-empirical models for predicting the noise generated by the main sources in the turbofan engine are used. The sound pressure levelsL(f) of the noise of the turbojet and turbofan resulting from each of $\mathrm{j}$ sources (where $\mathrm{j}=1$ represents the coaxial jets, $\mathrm{j}=2$ represents the fan, $j=3$ represents the turbine, $j=4$ represents the combustion chamber, and $\mathrm{j}=5$ represents the airframe) in each thirdoctave frequency band with center frequencyf are predicted by the simple relationship

$L_{j}(f)=L_{j}(\lambda)+Y_{j}$

where $\lambda$ is the vector of parameters used in the jth source model (forexample, velocity, temperature of the primary and the secondary of coaxialjets), and $\boldsymbol{Y}_{j}$ is an adjustment vector determined from the jth sourcemodel ${ }^{24}$.

The sound pressure levels due to coaxial shock-free jets without correction for refraction in the far field and effects of atmospheric absorption are calculated from:

$L_{1}\left(f_{i}, \lambda\right)=10 \log \left(\frac{A_{2}}{s^{2}}\right)+20 \log \left(\frac{g_{A}}{g_{s A}}\right)+\sum_{i} \Delta L_{i}(f, \lambda)+Y_{2}$

Where $\mathrm{A}_{1}$ is the area of the exit jet,ris the source-to-observer distance, $p_{\mathrm{A}}, p_{\mathrm{SA}}$ are respectively the ambient and standard sea level atmosphere pressuresand $\Delta \mathrm{L}_{1}(\mathrm{f}, \boldsymbol{\lambda})$ are spectral corrections for coaxial jets. As a rule, a referencedistance of 1 $\mathrm{m}$ is adopted. $\Delta \mathrm{L}_{11}(\mathrm{f}, \lambda)$, the correction of the noise level forreference base turbojet, is a function of the jet Mach number and polarangle from the inlet axis. $\Delta \mathrm{L}_{12}(\mathrm{f}, \boldsymbol{\lambda})$, the additive spectral correction is afunction of the jet Mach number, of the polar angle from inlet centerlineto exhaust centerline and of the jet temperature and density. $\Delta \mathrm{L}_{13}$ (f, $\lambda$ ), theflight velocity correction, is a function of the aircraft flight velocity, jet Machnumber and polar angle from the inlet axis. $\Delta \mathrm{L}_{14}$ ( $\left.\mathrm{f}, \lambda\right)$, the correction onthe coaxial jets, is a function of the primary and secondary velocity, density,temperature of airflow, of the exit jets diameters and of the polar angle fromthe inlet axis. The functions $\Delta \mathrm{L}_{1 \mathrm{i}}(\mathrm{f}, \boldsymbol{\lambda})$ are determined by choosing values ofkey parameters (for example, Strouhal and Mach number, the enthalpy ratio,the density ratio, geometrical parameters) that provide minimum differencesbetween experimentally measured and calculated values of the jet soundpressure level given by [15].

\section{2) Fan and turbine noise}

The fan, compressor and turbine of an aircraft engine generate tonal and broadband noise. Broadband noise results from the interaction of inhomogeneous pressure with turbulent flow. The blade-passage tone and its harmonics for subsonic tip Mach numbers result from the interactions of the pressure fields produced by the flow on the rotor/stator blade rows. There are additional multiple pure tones - 'buzz-saw' noise which accompany supersonic tip Mach numbers associated with supersonic flow on the blades and the formation of shock waves. The latter phenomenon is typical during takeoff. To determine the acoustical characteristics of the fan, compressor and turbine, it is necessary to take account of the noise generation, the noise propagation in the duct and the forward acoustic radiation, the rearward radiation from the bypass duct and core of the engine.

The sound pressure levels of the fan (or turbine) are given by equation (3), excluding the effects of atmospheric absorption and assuming that there is no acoustic treatment:

$$
L_{z}\left(f_{i} \lambda\right)=10 \log \left(\frac{A_{2}}{L_{2}}\right)+\sum_{i} \Delta L_{z i}\left(f_{i} \lambda\right)+Y_{z}(i=1 \text { to } 10)
$$

Where $\mathrm{A}_{2}$ is the inlet flow area for the fan (which is the exit flow area for the turbine); $\Delta \mathrm{L}_{21}(\boldsymbol{\lambda})$ and $\Delta \mathrm{L}_{22}(\boldsymbol{\lambda})$ are, respectively, the noise level corrections determined by the temperature difference $\Delta$ Tbetween the fan and the turbine; $\Delta \mathrm{L}_{23}(\mathrm{f}, \lambda)$ is the correction for multiple pure tones; $\Delta \mathrm{L}_{24}(\mathrm{f}, \boldsymbol{\lambda})$ and $\Delta \mathrm{L}_{25}(\mathrm{f}, \lambda)$ are, respectively, the spectral corrections for the broadband and tonal noise sources considered as a function of the third-octave frequency band spectrumf; $\Delta \mathrm{L}_{26}(\lambda)$ is the correction for the tip-speed Mach number $\mathrm{M}_{t} ; \Delta \mathrm{L}_{27}(\mathrm{f}, \lambda)$, $\Delta \mathrm{L}_{28}(\mathrm{f}, \lambda)$ are, respectively, corrections for the directivity of the broadband and tonal noise sources considered as function of the polar angle from inlet centerline to exhaust centerline $\theta$; $\Delta \mathrm{L}_{29}(\mathrm{f}, \lambda)$ is the flight velocity correction considered as a function of the aircraft flight velocity; and $\Delta \mathrm{L}_{2,10}(\boldsymbol{\lambda})$ is a 
correction depending on the peculiarity of the turbofan (turbine) construction (rotor-stator axial spacing, type of the mixed-flow exhaust nozzle, vane-blade ratio and so on) [15].

\section{FEEDFORWARD NEURAL NETWORKS}

In feedforward neural networks artificial neurons (also called nodes or processing units) are arranged in a feedforward manner (usually in the form of layers, i.e. each neuron may receive an input from the external environment and/or from other neurons, but no feedback is formed. A standard feedforward neural network consists of simple processing units (without dynamic elements). A feedforward network computes an output pattern in response to some input pattern. Once trained (with fixed connection weights) the output response to a given input pattern will be the same regardless of any previous network activity. This means that the feedforward neural network does not exhibit any real dynamics, and there are no stability problems in such networks. For feedforward networks the dynamics are often simplified to a single instantaneous nonlinear mapping. Some learning algorithm of the ANN can be described in the following $[16,17]$;

\section{A. Quickpropagation (QP) learning algorithm}

QP is another training method based on the following assumptions, E(w) for each weight can be approximated by a parabola that opens upward and the change in slope $E(w)$ fort his weight is not affected by other weights that change at the same time. The weight update rule is;

$$
\Delta w(t)=\frac{s(t)}{s(t-1)-s(t)} \Delta w(t-1)-\eta S(t)
$$

The numerator is the derivative of the error with respect to the weight and is a finite difference approximation of the second derivative. Together these approximate Newton's method for minimizing a one-dimensional function . To avoid an infinite backward step, or a backward uphill step, a maximum growth factor parameter $\mu$ is introduced. No weight change is allowed to be larger than $\mu$ times the previous weight change. Furthermore, QP has a fixed learning parameters, that needs to e chosed to suit the problem.

\section{B. Delta-Bar-Delta (DBD) learning algorithm}

An adaptive learning rate method in which every weight has its own learning rate. The learning rates are updated based on the sign of the gradient does not change signs on successive iterations then the step size is increased linearly. If the gradient changes signs, the learning rate is decreased exponentially. In some cases this method seems to learn much faster than non-adaptive methods. Learning rates $(t)$, are updated as follows;

$$
\Delta_{i j}\left\{\begin{array}{lr}
\kappa & \text { if } \sigma^{\prime}(t-1) \sigma(t)>0 \\
-\phi \eta(t) & \text { if } \sigma^{\prime}(t-1) \sigma(t)<0 \\
0 & \text { else }
\end{array}\right.
$$

Where $\delta(\mathrm{t})=\frac{\partial E}{\partial W}$ at time $\mathrm{t}$ and $\delta$ is the exponential average of past values of $\delta . \delta^{\prime}(t)=(1-\theta) \delta(t)+\theta \delta^{\prime}(t-1)$

\section{SIMULATION RESULTS}

Simulation study has been carried out for predicting noises of Jet, Turbine and Fan of an aircraft system using neural network predcitors. Two types of learining algoritms were employed to find exact and robust perdictor for air craft system's noise analysis. Noise prediction prosess of the aircraft is described in detail in Figure 2. From the figure, $f$ is third-octave band center frequency $[\mathrm{Hz}] . P_{s}$ is sound pressure level $[\mathrm{dB}], P_{S J}$ is sound pressure level $[\mathrm{dB}], P_{s F}$ sound pressure level of jet $[\mathrm{dB}]$ and $P_{s T}$ is sound pressure level of turbine $[\mathrm{dB}] . P_{S J N N}$ is sound pressure level $[\mathrm{dB}]$ output of the neural network, $P_{s f N N}$ sound pressure level of jet $[\mathrm{dB}]$ output of the neural network and $P_{S T N N}$ is sound pressure level of turbine $[\mathrm{dB}]$ output of the neural network.

Therefore, a third-octave frequency band spectrum and an overall sound pressure level (OASPL) for an aircraft with a low bypass engine $(\mathrm{m}=1)$ during takeoff engine mode measured at the lateral noise monitoring point $1(450 \mathrm{~m}$ from the runway axis) are measured and shown in Fig. 3 for the case of 1. Figure 4 shows the measured noise characteristics of the same aircraft at the flyover noise monitoring measurement point $(6500 \mathrm{~m}$ from aircraft gear release on runway during takeoff) for the case of 2 . The engine mode is nominal.Noise source contributions for aircraft with low bypass ratio engines (bypass engine ratio, $m=1$ ) at control point (takeoff mass $160 \mathrm{t}$, distance $450 \mathrm{~m}$, engine mode at maximum thrust, 'lateral attenuation' neglected).

Another approaches of the proposed neural netwok predictor for the case of 3 is described with the graph of Figure 5. From the figure, turbine noises seem stable for the case of 3 . But fan and jet noises are at the high level $(74 \mathrm{~dB})$.

Moreover, at present, attention is focused mainly on the noise reduction of engines with high bypass ratios $(m \geq 6)$, since they are widely used. Consideration is given to possible design methods: optimization of fan, gas-dynamic and operation parameters on the basis of integrated aeroacoustic design and installation of intake and exhaust silencers.

Again, a third-octave frequency band spectrum and an overall sound pressure level (OASPL) for an aircraft with a low bypass engine $(\mathrm{m}=1)$ during takeoff engine mode measured at the lateral noise monitoring point $1(450 \mathrm{~m}$ from the runway axis) are measured and shown in Fig. 6.The results described other neural predictor approach with delta-bar-delta learning algorithm for the case of 4 . As can be seen from the 
graph, this approach is not exect match experimental approach.

The case of 5 is shown in Figure 7. The figure is indicated that fan noises are increased suddenly until 2000 third-octave central band frequency. After this frequency, it is behaviour stable. Turbine and jet noises are at the same on 5500 thirdoctave central band frequency.

Neural network prediction approaches for jet, turbine and fan noises of aircraft are shown in Figure 8. From the graph, neural predictor approach is following the experimental measured data set.

However, table 1. shows training, structural and RMSE (root mean square error) parameteres of the neural network for the cases of 1-6.

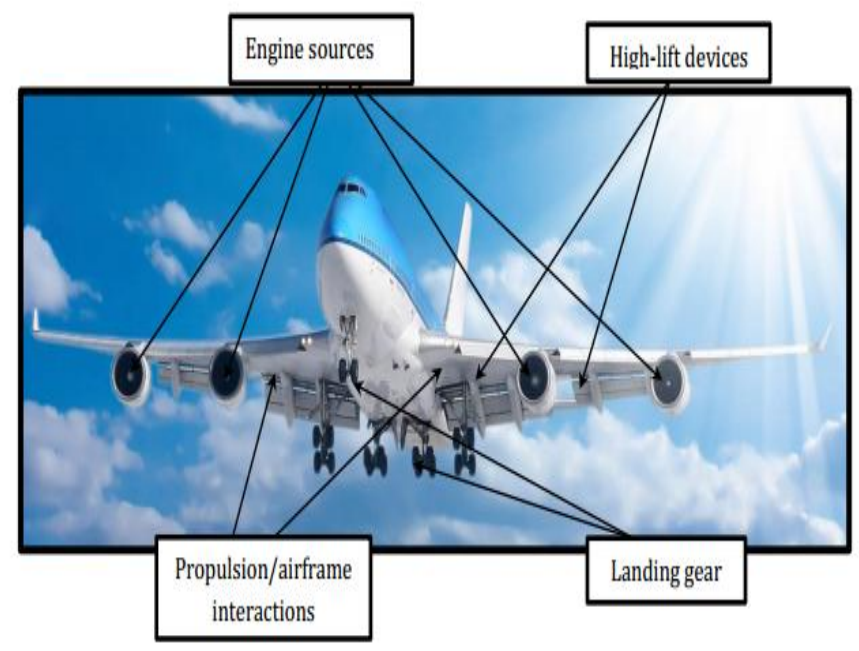

Fig. 1. Aircraft noise sources.

Table I. STRUCTURAL ANDTRAININGPARAMETERS OFNEURAL PREDICTOR

\begin{tabular}{|c|c|c|c|c|c|}
\hline Cases & Learning Algorithm & $\begin{array}{c}\text { Learning } \\
\text { rate }\end{array}$ & Networktype & $\begin{array}{c}\text { Training } \\
\text { numbers }\end{array}$ & RMSE \\
\hline Case-1- & Quick Propgation & 0.1 & $1 \cdot 10-3$ & 5000000 & 0.151284 \\
\hline Case-2- & QuickPropagation & 0.1 & $1 \cdot 10 \cdot 3$ & 5000000 & 1.619150 \\
\hline Case-3- & QuickPropagation & 0.1 & $1 \cdot 10 \cdot 3$ & 5000000 & 0.306890 \\
\hline Case-4· & Delta-Bar-Delta & 0.1 & $1 \cdot 10-3$ & 5000000 & 0.617833 \\
\hline Case-5· & Delta-Bar-Delta & 0.1 & $1 \cdot 10-3$ & 5000000 & 3.056930 \\
\hline Case-6- & Delta-Bar-Delta & 0.1 & $1 \cdot 10-3$ & 5000000 & 1.163710 \\
\hline
\end{tabular}

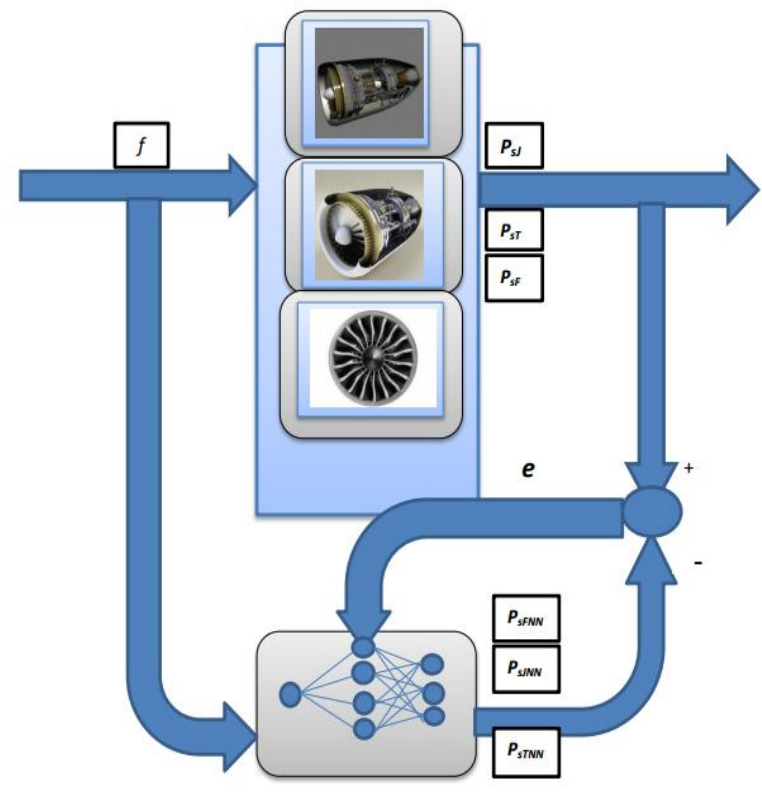

Fig. 2. Description of system analysis

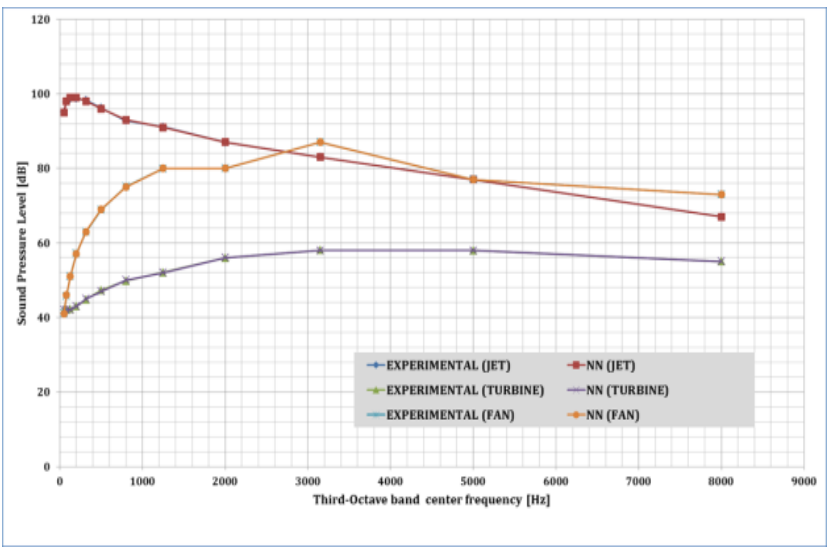

Fig. 3. Neural network prediction approaches for jet, turbine and fan noises of aircraft (Case 1) 


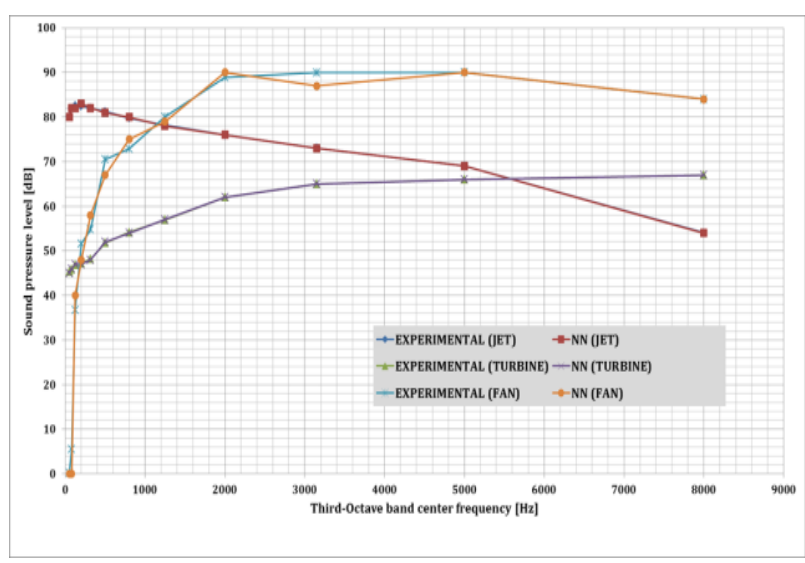

Fig. 4. Neural network prediction approaches for jet, turbine and fan noises of aircraft (Case 2)

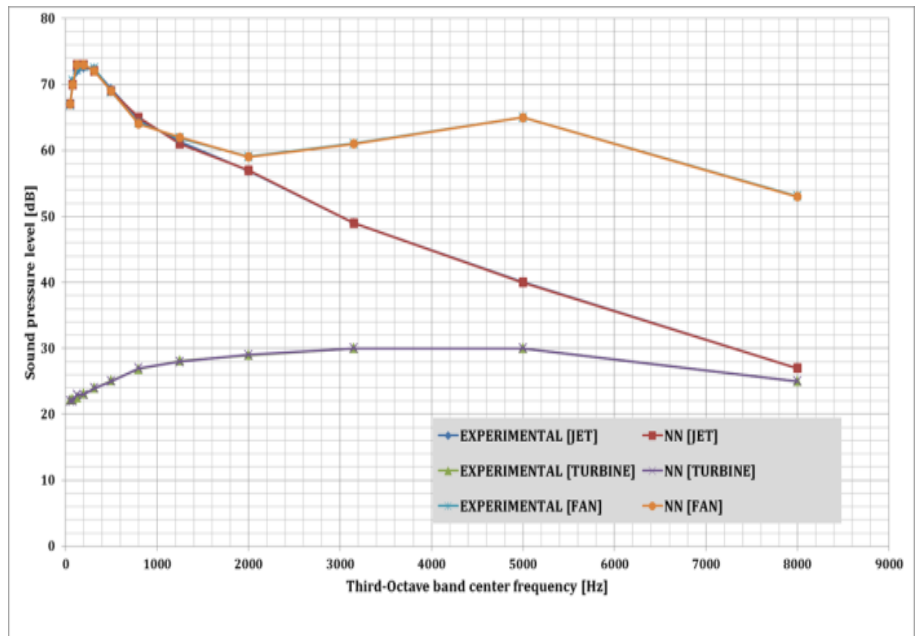

Fig. 5. Neural network prediction approaches for jet, turbine and fan noises of aircraft (Case 3)

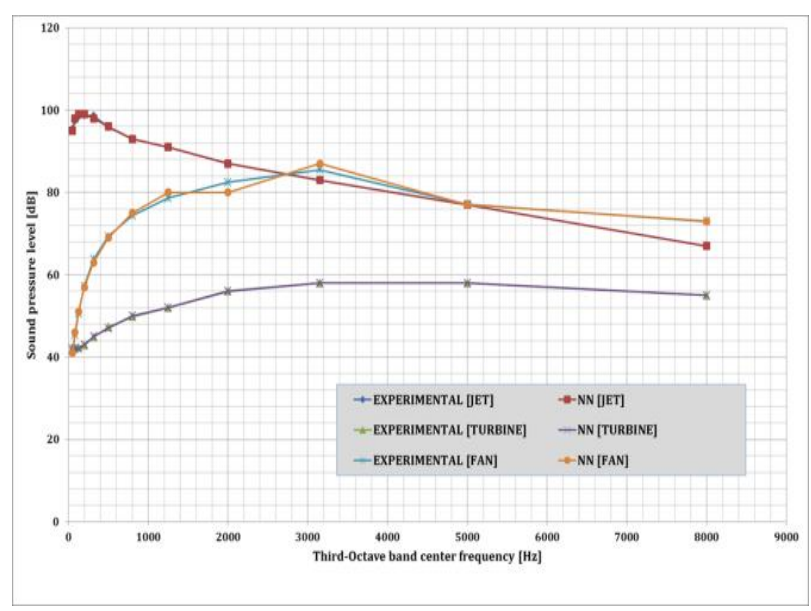

Fig. 6. Neural network prediction approaches for jet, turbine and fan noises of aircraft (Case 4)

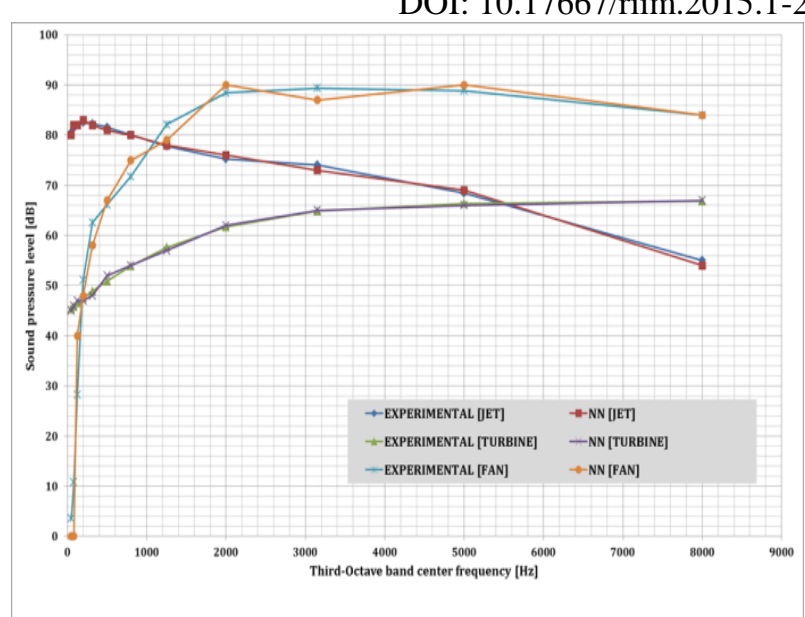

Fig. 7. Neural network prediction approaches for jet, turbine and fan noises of aircraft (Case 5)

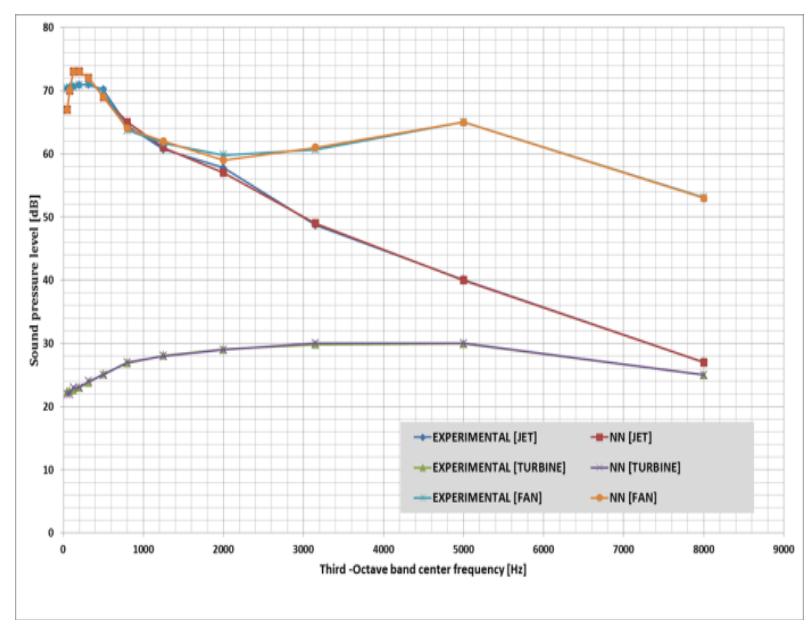

Fig.8. Neural network prediction approaches for jet, turbine and fan noises of aircraft (Case 6)

\section{CONCLUSION AND DISCUSSION}

There has been a considerable decrease in noise levels from individual aircraft over the past 35 years. The noise levels produced by modern aircraft are about $22 \mathrm{~dB}$ lower than those of first generation jet aircraft. This reduction has been achieved as a result of the development of turbofan engines with high bypass ratios, liner technology and turbomachinery source noise reduction. Further aircraft noise reduction will be achieved from improved design of engine systems, decrease in airframe sources and the introduction of noise reduction technologies. The acoustic model of an aircraft is the sum of particular models for the various noise sources including jet (propeller), fan (compressor), combustion chamber, turbine and airframe. They enable assessment of the aircraft as a complex noise source and investigation of the influence on the overall aircraft acoustic design of powerplant parameters. 
To predict unwanted disturbances such as wind, storm etc. a neural network based predictor has been used to predict noises of the various noise sources including jet (propeller), fan (compressor), combustion chamber, turbine and airframe. The proposed neural predictors have performance to adapt unpredicted noise sources.

Neverthless, jet noise reduction techniques are based on reducing noise emission with minimal loss of jet thrust (less than between 3 and 5 per cent). The propeller is the main noise source on a turboprop engine. Propeller noise arises as a result of the periodic displacement of the air by the volume of a passing blade (thickness noise). The pressure fluctuation due to lift and draft disturbance gives the loading noise of the propeller.

\section{References}

[1] T. Callum, (2000) "Environmental capacity of airports - what does it mean?' Workshop Proceedings 2 Environmental Capacity” The challenge for aviation industry. London, Heathrow Airport, pp. 8$11,2000$.

[2] M. Janic, "Modeling operational, economic and environmental performance of an air transport network",Transportation Research,8, pp. 415-32, 2003.

[3] P.M. Nelson, (ed.)"Transportation Noise Reference Book", London: Butterworths \& Co. Ltd, 1987.

[4] V.E. Kvitka, B. N. Melnikov, and V.I. Tokarev, "Standardization and Noise Abatement for Airplanes and Helicopters"[in Russian]. Kyiv, Vyscha Shkola, 1980.

[5] Zaporozhets, O., Tokarev, V. and Attenborough, K., Aircraft Noise Assessment, prediction and control, Spon Press, New York, NY, USA, 2011.

[6] Recommended Method for Computing Noise Contours around Airports, Circular 205 ICAO, International Civil Aviation Organization, 1987.

[7] Standard Method of Computing Noise Contours around Civil Airports, ECAC Document 29. 1997.

[8] Procedure for the Calculation of Airplane Noise in the Vicinity of Airports, Society of Automotive Engineers, SAE AIR 1845, 1986.

[9] Integrated Noise Model (INM) version 6.0, Report No. FAAAEE-99-03, Washington, Federal Aviation Administration, 2000.

[10] The UK Civil Aircraft Noise Contour Model ANCON: Improvements in Version 2, National Air Traffic Services, 1999.

[11] Nord 2000. Comprehensive Outdoor Sound Propagation Model, 2001.

[12] Prediction method for lateral attenuation of airplane noise during takeoff and landing. AIR 1751 (1981), Society of Automotive Engineers. Inc.

[13] Gas turbine jet exhaust noise prediction. ARP 876C (1982) Society of Automotive Engineers. Inc.

[14] House, M.E. and Smith, M.J.T. "Internally generated noise from gas turbine engines - measurement and prediction", ASME Paper 66-GT/N-43, 1966.

[15] Gas turbine coaxial exhaust flow noise prediction. AIR 1905 , Society of Automotive Engineers. Inc. 1985.

[16] S. Yıldırım, and İ. Eski, "Noise Analysis Of Robot Manipulator Using Neural Networks", Robotics and Computer-Integrated Manufacturing, 26, pp.282-290, 2010.

[17] S. Yıldırım, "Vibration Control of Suspensions Using a Proposed Neural Network", Journal of Sound and Vibration, vol. 27, pp. 1059-1069, 2004. 